$\xi=-$ 圆

\title{
An M/M/1 Queue with Working Vacation and Vacation Interruption Under Bernoulli Schedule
}

\author{
P. Manoharan* and A. Ashok \\ Department of Mathematics, \\ Annamalai University, Annamalai Nagar, -608 002 \\ *Corresponding author E-mail: manomaths.hari@gmail.com
}

\begin{abstract}
This work deals with M/M/1 queue with Vacation and Vacation Interruption Under Bernoulli schedule. When there are no customers in the system, the server takes a classical vacation with probability $\mathrm{p}$ or a working vacation with probability $1-\mathrm{p}$, where $0 \leq p \leq 1$. At the instants of service completion during the working vacation, either the server is supposed to interrupt the vacation and returns back to the non-vacation period with probability 1-q or the sever will carry on with the vacation with probability $\mathrm{q}$. When the system is non empty after the end of vacation period, a new non vacation period begins. A matrix geometric approach is employed to obtain the stationary distribution for the mean queue length and the mean waiting time and their stochastic decomposition structures. Numerous graphical demonstrations are presented to show the effects of the system parameters on the performance measures.
\end{abstract}

Keywords: M/M/1 queue Working vacation Vacation Vacation Interruptions Matrix-geometric solution.

\section{Introduction}

Queuing Models under vacation policy have been studied extensively with regard to their application in computer, computer systems and production management. Numerous vacation policy enrich the adaptability for optimal design of the queuing systems. Queuing models with server vacations or working vacation have been studied by various researchers during the last two decades. The literature may be divided into two groups (i) when the server is on a vacation and (ii) when the server is on a working vacation. When the server is on a vacation, the survey paper by Doshi (1986) and the monograph of Takagi (1991) act as the reference for the readers. The research work done by Takagi (1991) and Doshi (1986) stresses on a single server. As far as the case of multiple-server system with vacations is concerned, Levy and Yechiali (1976) first studied the $M / M / c$ queue with asynchronous vacation policy. Later, Chao and Zhao (1998) analyzed the $M / M / c$ with both synchronous and asynchronous vacation policies and computed the stationary probability distribution by providing some algorithms. Zhang and Tian (2003a, 2003b) recently contributed enough analysis of $M / M / c$ queue with synchronous multiple/single vacations of partial servers.

$M / M / 1$ queuing models with multiple working vacations was first studied by Servi and Finn (2002), where inter-arrival times, service times during service period, service times during vacation period and vacation times are all exponentially distributed (Such model is denoted by $M / M / 1 / W V$ queue). Later $M / M / 1 / W V$ model was also studied by Liu et al. (2007) to obtain explicit expressions of the performance measures and their stochastic decomposition by using the quasi-birth death and matrix-geometric method. Servi and Finn (2002) queue extended to $M / G / 1 / W V$ queue by $\mathrm{Wu}$ and Takagi (2006). They assumed that service times during service period, service times during vacation period as well as vacation times are all generally distributed. Further, they assumed that at the end of the working vacation, if the system has customers, the server switches to another service rate, where a different distribution is followed by the service times. The $G I / M / 1 / W V$ queue is the advanced form of Servi and Finn (2002), extended by Baba (2005). Not only assuming general independent arrival, they also considered service times during service period, service times during vacation period as well as vacation times following exponential distribution. The analysis of single working vacation in $G I / M / 1 / N$ and $G I / M / 1 / 1$ queuing systems was done by Banik (2010) by assuming that the service time and vacation time was distributed exponentially. Laxmi and Yesuf (2011) recently presented an $G I / M / 1$ batch service queue with a policy of exponential working vacation. Lin and $\mathrm{Ke}$ (2009) analyzed the $M / M / c$ queue with single working vacation. A short survey on recent developments in vacation queuing models has been also given by Ke et al. (2010).

Generally, in working vacation policy, the server starts again his work at regular service rate after the end of vacation, only if the customers are waiting at the system. Definitely such speculations appears much more limited in real world situations. To come out of this restriction, $\mathrm{Li}$ and Tain [1] introduced the vacation interruption schedule in an $\mathrm{M} / \mathrm{M} / 1$ queue with working vacations. In this vacation policy, a server at the completion of vacation instantly ends his vacation and comes back to its normal working level if customers are waiting in the queue, instead he will continue his vacation till the system is non empty after vacation ends. Due to its strong application in the stochastic service models, it gives productive theoretical results in this area. Li et al. [2], 
Baba [3], Zhang and Hou [4], Gao and Liu [5], and Lee and Kim [6] are those who gave eminent papers in this area.

This paper is organized as follows. In section 2, we present the discription of the model and discuss the model as quasi birth and death process. In section 3 , we derived the stationary distribution of the queue length and the state probabilities of the server.

Section 4, we present the stochastic decomposition structures of the stationary queue length and Sojourn time. Finaly, numerical demonstrations are given in section 5 .

Model description: We consider an M/M/1 queueing system where the customers arrive according to Poisson process with rate $\lambda$. The server begins to serve the customers at a rate $\mu$ which is exponentially distributed. When there are no customers in the system, the server takes a classical vacation with probability $\mathrm{p}$ or a working vacation with probability $1-\mathrm{p}$, where $0 \leq p \leq 1$. During the working vacation, customers are served at an exponential rate $\eta$ where $\eta<\mu$ i.e. the customer is served at a reduced service rate. Moreover, at the instants of service completion during the working vacation, either the server is supposed to interrupt the vacation and returns back to the non-vacation period with probability $1-\mathrm{q}$ or the sever will carry on with the vacation with probability q. When the system is non empty after the end of vacation period, a new non vacation period begins. The classical vacation times and the working vacation times are also assumed to be exponentially distributed with parameters $\theta$ and $\gamma$, respectively.

Let $\mathrm{Q}(\mathrm{t})$ be the number of customers in the system at time $t$ and $\mathrm{J}(\mathrm{t})$ be the status of the server, which is defined as follows

$J(t)= \begin{cases}0, & \text { when the server is in working vacation period at time t, } \\ 1, & \text { when the server is in classical vacation at time } \mathrm{t}, \\ 2, & \text { when the server is in non-vacation period at time } \mathrm{t} .\end{cases}$

Then $\{(Q(t), J(t)), t \geq 0\}$ defines a Markov Process with continuous time state space

$S=\{(k, j): k=0,1,2, \ldots, j=0,1\}$

Using the lexicographical sequence for the states, the infinitesimal generator can be written as

$Q=\left(\begin{array}{cccccc}B_{0} & A_{0} & & & & \\ C_{0} & B & A & & & \\ & C & B & A & & \\ & & C & B & A & \\ & & & \vdots & \vdots & \vdots \\ & & & & & \end{array}\right)$

Where $B_{0}=\left(\begin{array}{cc}-\lambda & 0 \\ 0 & -\lambda\end{array}\right), A_{0}=\left(\begin{array}{ccc}\lambda & 0 & 0 \\ 0 & \lambda & 0\end{array}\right)$,

$C_{0}=\left(\begin{array}{cc}\mu_{v} & 0 \\ 0 & 0 \\ (1-p) \mu_{b} & p \mu_{b}\end{array}\right)$,

$B=\left(\begin{array}{ccc}-\left(\lambda+\mu_{v}+\gamma\right) & 0 & \gamma \\ 0 & -(\lambda+\theta) & \theta \\ 0 & 0 & -\left(\lambda+\mu_{b}\right)\end{array}\right), n \geq 1$

$A=\left(\begin{array}{ccc}\lambda & 0 & 0 \\ 0 & \lambda & 0 \\ 0 & 0 & \lambda\end{array}\right), \quad C=\left(\begin{array}{ccc}q \mu_{v} & 0 & (1-q) \mu_{v} \\ 0 & 0 & 0 \\ 0 & 0 & \mu_{b}\end{array}\right)$
The matrix structure of $\mathrm{Q}$ indicates that the Markov Chain $\{(Q(t), J(t)), t \geq 0\}$ is a quasi birth and death process. To analyze the QBD process, we need to obtain the minimal non negative solution of the matrix quadratic equation which is said to be the rate matrix and it is denoted by $R$,

$$
R^{2} B+R A+C=0
$$

The following lemma presents the explicit solution of $\mathrm{R}$.

\section{Lemma 1.}

If $\rho=\frac{\lambda}{\mu}<1$, the minimal non-negative solution of matrix quadratic equation (1) has the following expression

$R=\left(\begin{array}{lll}r & 0 & \alpha \\ O & \beta & \rho \\ O & 0 & \rho\end{array}\right)$

Where

$$
\begin{aligned}
& r=\frac{\left(\lambda+\mu_{v}+\theta\right)-\sqrt{\left(\lambda+\mu_{v}+\theta\right)^{2}-4 q \mu_{v} \lambda}}{2 q \mu_{v}}, 0<r<1 \\
& \alpha=\frac{r\left[(1-q) \mu_{v} r+\gamma\right]}{\mu_{b}(1-r)} \\
& \beta=\frac{\lambda}{\lambda+\theta}
\end{aligned}
$$

\section{Proof.}

Since A, B and C of (1) are all upper triangular matrices, we can consider that the solution matrix $\mathrm{R}$ has the same structure as

$R=\left(\begin{array}{ccc}r_{11} & r_{12} & r_{13} \\ 0 & r_{22} & r_{23} \\ 0 & 0 & r_{33}\end{array}\right)$

Substituting the value of $R^{2}$ and $\mathrm{R}$ into (1) leads to

$$
\begin{aligned}
& q \mu_{v} r_{11}^{2}-\left(\lambda+\mu_{v}+\gamma\right) r_{11}+\lambda=0 \\
& \mu_{b} r_{33}^{2}-\left(\lambda+\mu_{b}\right) r_{33}+\lambda=0 \\
& \lambda-(\lambda+\theta) r_{22}=0 \\
& (1-q) \mu_{v} r_{11}^{2}+\left(r_{11} r_{13}+r_{13} r_{33}\right) \mu_{b}+\gamma r_{11} \\
& -\left(\lambda+\mu_{b}\right) r_{13}=0 \\
& r_{22} \theta-\left(\lambda+\mu_{b}\right) r_{23}+\mu_{b}\left(r_{22} r_{23}+r_{23} r_{33}\right)=0
\end{aligned}
$$

From the above set of equation we can obtain the minimal nonnegative solution of (1) by using the fact that (3) has a unique solution $r$

$r=\frac{\left(\lambda+\mu_{v}+\theta\right)-\sqrt{\left(\left(\lambda+\mu_{v}+\theta\right)^{2}-4 q \mu_{v} \lambda\right.}}{2 q \mu_{v}}$ 
in interval $(0,1)$. Substituting $r_{11}=r$ and $r_{33}=\rho$ into (6), we get $r_{13}=\frac{r\left[(1-q) \mu_{v} r+\gamma\right]}{\mu_{b}(1-r)}=\alpha$.Substituting the value $r_{22}=\frac{\lambda}{\lambda+\theta}=\beta$ and $r_{33}=\rho$ into (7), we get $r_{23}=\rho$

\section{Theorem 1.}

The Quasi Birth-Death process $\{Q(t), J(t)\}$ is positive recurrent if and only if $\rho<1$.

\section{Proof.}

Based on the lemma.1 of neuts (1981) the Quasi Birth death process $\{Q(t), J(t)\}$ is positive recurrent if and only if the spectral radius $\mathrm{SP}(\mathrm{R})$ of the rate matrix $\mathrm{R}$ is less than 1 , and set of equation $\left(x_{0}, x_{1}, x_{2}\right) B[R]=0$ has positive solution where

$$
\begin{aligned}
& B[R]=\left[\begin{array}{cc}
B_{0} & A_{0} \\
C_{0} & R C+B
\end{array}\right] \\
& =\left[\begin{array}{ccccc}
-\lambda & 0 & \lambda & 0 & 0 \\
0 & -\lambda & 0 & \lambda & 0 \\
\mu_{v} & 0 & -\left(\lambda+\mu_{v}(1-r q)+\gamma\right) & 0 & r(1-q) \mu_{v}+\alpha \mu_{b}+\gamma \\
0 & 0 & 0 & -(\lambda+\theta) & (\lambda+\theta) \\
(1-p) \mu_{b} & p \mu_{b} & 0 & 0 & -\mu_{b}
\end{array}\right]
\end{aligned}
$$

$\mathrm{B}[\mathrm{R}]$ is an irreducible and aperiodic generator with finite state. Therefore $\left(x_{0}, x_{1}, x_{2}\right) B[R]=0$ has positive solution (for example, the balance probability vector of $\mathrm{B}[\mathrm{R}]$ is positive solution). Thus, process $\{Q(t), J(t)\}$ is positive recurrent if and only if

$$
S P(R)=\max (r, \rho)<1
$$

Stationary distribution of queue length: If $\rho<1$, let $(\mathrm{Q}, \mathrm{J})$ be the stationary limit of the QBD process $\{(Q(t), J(t)), t \geq 0\}$ and define

$$
\begin{aligned}
& \pi_{0}=\left(\pi_{00}, \pi_{01}\right) \\
& \pi_{k}=\left(\pi_{k 0}, \pi_{k 1}, \pi_{k 2}\right), \quad k \geq 1 \\
& \pi_{k j}=P\{Q=k, J=j\} \quad k \geq 0, \quad J=0,1
\end{aligned}
$$

\section{Theorem 2.}

If $\rho<1$, the Stationary probability distribution of $(\mathrm{Q}, \mathrm{J})$ is

$$
\begin{aligned}
\pi_{k 0}= & \frac{\lambda K}{\lambda+\mu_{v}(1-r q)+\gamma} r^{k-1}, k \geq 0, \\
\pi_{k 1}= & \frac{\lambda p K\left(\lambda-q r \mu_{v}+\gamma\right)}{(\lambda+\theta)(1-p) \lambda+\mu_{v}(1-r q)+\gamma} \beta^{k-1}, k \geq 0 \\
\pi_{k 2}= & \frac{\lambda K \alpha}{\lambda+\mu_{v}(1-r q)+\gamma} \sum_{j=0}^{k-1} \rho^{j} r^{k-1-j} \\
& +\frac{p K\left(\lambda-q r \mu_{v}+\gamma\right)}{(1-p)\left(\lambda+\mu_{v}(1-r q)+\gamma\right)} \rho \sum_{j=0}^{k-1} \rho^{j} \beta^{k-1-j} \\
& -\frac{\lambda K \alpha \rho-1}{r\left(\lambda+\mu_{v}(1-r q)+\gamma\right)}+\frac{K\left(\lambda-q r \mu_{v}+\gamma\right) \rho}{\left(\lambda+\mu_{v}(1-r q)+\gamma\right)}, k \geq 1
\end{aligned}
$$

where

$$
K=\frac{\lambda+\mu_{v}(1-r q)+\gamma}{\left[\frac{\lambda}{r(1-r)}+\frac{p\left(\lambda-q r \mu_{v}+\gamma\right)}{(1-p)(1-\beta)(1-\rho)}+\frac{\lambda \alpha}{(1-\rho)(1-r)}+\frac{\left(\lambda-q r \mu_{v}+\gamma\right) \rho}{1-\rho}\right]}
$$

Proof.

Using the matrix geometric solution method by Neuts (1981), we get

$$
\pi_{k}=\left(\pi_{k 0}, \pi_{k 1}, \pi_{k 2}\right)=\left(\pi_{10}, \pi_{11}, \pi_{12}\right) R^{k-1}, \quad k \geq 1
$$

and $\left(\pi_{00}, \pi_{01}, \pi_{10}, \pi_{11}, \pi_{12}\right)$ satisfies the following set of equations

$\left(\pi_{00}, \pi_{01}, \pi_{10}, \pi_{11}, \pi_{12}\right) B[R]=0$

Substituting $B[R]$ in (8) into the above relation, we have

$$
\begin{gathered}
-\lambda \pi_{00}+\mu_{v} \pi_{10}+(1-p) \mu_{b} \pi_{12}=0 \\
-\lambda \pi_{01}+p \mu_{b} \pi_{12}=0 \\
\lambda \pi_{00}-\left(\lambda+\mu_{v}(1-r q)+\gamma\right) \pi_{01}=0 \\
\lambda \pi_{01}-(\lambda+\theta) \pi_{11}=0 \\
\left(r(1-q) \mu_{v}+\alpha \mu_{b}+\gamma\right) \pi_{10}+(\lambda+\theta) \pi_{11}=0
\end{gathered}
$$

Taking $\pi_{00}=K$ and solving the above equations in terms of $\mathrm{K}$, we obtain

$$
\begin{aligned}
& \pi_{01}=\frac{p K\left(\lambda-q r \mu_{v}+\gamma\right)}{(1-p)\left(\lambda+(1-q r) \mu_{v}+\gamma\right)} \pi_{10}=\frac{\lambda K}{\left(\lambda-q r \mu_{v}+\gamma\right)} \\
& \pi_{11}=\frac{\lambda p K\left(\lambda-q r \mu_{v}+\gamma\right)}{(\lambda+\theta)(1-p)\left(\lambda+(1-q r) \mu_{v}+\gamma\right)}, \\
& \pi_{12}=\frac{\lambda K\left(\lambda-q r \mu_{v}+\gamma\right)}{(1-p) \mu_{b}\left(\lambda+(1-q r) \mu_{v}+\gamma\right)}
\end{aligned}
$$

From (2), we obtain

$$
R^{k}=\left[\begin{array}{ccc}
r^{k} & 0 & \alpha \sum_{j=0}^{k-1} \rho^{j} r^{k-1-j} \\
0 & \beta^{k} & \rho \sum_{j=0}^{k-1} \rho^{j} \beta^{k-1-j} \\
0 & 0 & \rho^{k}
\end{array}\right], \quad k \geq 1
$$

Similarlly,

$$
R^{k-1}=\left[\begin{array}{ccc}
r^{k-1} & 0 & \alpha \sum_{j=0}^{k-2} \rho^{j} r^{k-2-j} \\
0 & \beta^{k-1} & \rho \sum_{j=0}^{k-2} \rho^{j} \beta^{k-2-j} \\
0 & 0 & \rho^{k-1}
\end{array}\right], \quad k \geq 1
$$

Substituting $\left(\pi_{10}, \pi_{11}\right)$ and the matrix expression $R^{k-1}$ into (10), we obtain (9) and noting that constant factor K can be determined by utilizing the normalization condition.

The probabilities of the server in various state are as follows, respectively. 
$P\{\mathrm{~J}=0\}=P\{$ The server is in working vacation period $\}$

$$
=\sum_{k=0}^{\infty} \pi_{k 0}=\left(\frac{\lambda K}{\lambda+\mu_{v}(1-r q)+\gamma}\right) \frac{1}{r(1-r)}
$$

$P\{\mathrm{~J}=1\}=P\{$ The server is in classical vacation period $\}$

$$
=\sum_{k=1}^{\infty} \pi_{k 1}=\left(\frac{\lambda K p\left(\lambda-q r \mu_{v}+\gamma\right)}{(1-p)\left(\lambda+\mu_{v}(1-q r)+\gamma\right)}\right) \frac{1}{1-\beta}
$$

$P\{\mathrm{~J}=2\}=P\{$ The server is in non-vacation period $\}=\sum_{k=1}^{\infty} \pi_{k 2}$

$$
\begin{aligned}
& =\frac{K}{\left(\lambda+\mu_{v}(1-q r)+\gamma\right)}\left[\frac{\lambda \alpha}{r(1-r)(1-\rho)}\right. \\
& \left.+\frac{p \rho\left(\lambda-q r \mu_{v}+\gamma\right)}{(1-\beta)(1-\rho)(1-p)}-\frac{\lambda \alpha}{r(1-\rho)}+\frac{\rho\left(\lambda-q r \mu_{v}+\gamma\right)}{1-\rho}\right]
\end{aligned}
$$

Stochastic decompositions: To achieve a better comparison with the queuing models already existing, we often attempt to decompose the quantities of interest into various factors. In vacation queuing models, the stochastic decomposition structures plays a significant role and underlines the influence of system vacation on system performance indices such as mean queue length and mean sojourn times. For the system under investigation, we strive to do a similar decomposition.

\section{Theorem 3.}

If $\rho<1$ and $\mu_{b}>\mu_{v}$, the number of customers $\mathrm{Q}$ in system can be decomposed into the sum of two independent random variable $Q=Q_{0}+Q_{d}$, where $Q_{0}$ is the number of customers of a classic $M / M / 1$ queue in steady state and follows a gementric distribution with parameter $(1-\rho), Q_{d}$ is the additional number of customer has a modified geometric distribution

$Q_{d}(z)=\delta_{1}+\delta_{2} z+\delta_{3} \frac{1-r}{1-r z} z+\delta_{4} \frac{1-\rho}{1-\rho z} z$

where

$$
\begin{aligned}
& \delta_{1}=\left[\frac{\lambda}{r}+\frac{p\left(\lambda-q r \mu_{v}+\gamma\right)}{1-p}\right](1-r)(1-\beta), \\
& \delta_{2}=\left[\rho\left(\lambda-q r \mu_{v}+\gamma\right)-\frac{\lambda \alpha}{r}\right](1-r)(1-\beta) \\
& \delta_{3}=(1-\beta)\left[\frac{\lambda}{r}(r-\rho)+\frac{\lambda \alpha}{r}\right], \\
& \delta_{4}=(1-r)\left[\frac{p\left(\lambda-q r \mu_{v}+\gamma\right)(\beta-\rho)}{1-p}+\frac{\rho p\left(\lambda-q r \mu_{v}+\gamma\right)}{1-p}\right] \\
& K^{*}=\frac{K}{(1-r)(1-\beta)(1-\rho)\left(\lambda+\mu_{v}(1-r q)+\gamma\right)}
\end{aligned}
$$

\section{Proof.}

From (9), the probability generating function of Q can be written as

$$
\begin{aligned}
& Q(z)=\sum_{k=0}^{\infty} \pi_{k 0} z^{k}+\sum_{k=0}^{\infty} \pi_{k 1} z^{k}+\sum_{k=1}^{\infty} \pi_{k 2} z^{k} \\
& =K\left[\frac{\lambda}{r(1-r z)}+\frac{p\left(\lambda-q r \mu_{v}+\gamma\right)}{1-p} \frac{1}{\beta(1-\beta z)}\right. \\
& +\frac{\lambda \alpha z}{r(1-r z)(1-\rho z)}+\frac{p \rho\left(\lambda-q r \mu_{v}+\gamma\right)}{1-p} \\
& \frac{z}{(1-\rho z)(1-\beta z)}-\frac{\lambda z}{(1-\rho z)} \\
& \left.+\left(\lambda-q r \mu_{v}+\gamma\right) \frac{\rho z}{1-\rho z}\right] \\
& =\frac{1-\rho}{1-\rho z} K^{*}\left[\delta_{1}+\delta_{2} z+\delta_{3} \frac{1-r}{1-r z} z+\delta_{4} \frac{1-\rho}{1-\rho z} z\right]
\end{aligned}
$$

Where

$$
\begin{aligned}
& \delta_{1}=\left[\frac{\lambda}{r}+\frac{p\left(\lambda-q r \mu_{v}+\gamma\right)}{1-p}\right](1-r)(1-\beta), \\
& \delta_{2}=\left[\rho\left(\lambda-q r \mu_{v}+\gamma\right)-\frac{\lambda \alpha}{r}\right](1-r)(1-\beta) \\
& \delta_{3}=(1-\beta)\left[\frac{\lambda}{r}(r-\rho)+\frac{\lambda \alpha}{r}\right], \\
& \delta_{4}=(1-r)\left[\frac{p\left(\lambda-q r \mu_{v}+\gamma\right)(\beta-\rho)}{1-p}+\frac{\rho p\left(\lambda-q r \mu_{v}+\gamma\right)}{1-p}\right]
\end{aligned}
$$

we prove that $\delta_{1}+\delta_{2}+\delta_{3}+\delta_{4}=\left(K^{*}\right)^{-1}$

$$
\begin{aligned}
\left(K^{*}\right)^{-1}= & \frac{(1-r)(1-\beta)(1-\rho)\left(\lambda+\mu_{v}(1-r q)+\gamma\right)}{K} \\
= & (1-r)(1-\beta)(1-\rho)\left[\frac{\lambda}{r(1-r)}+\right. \\
& \frac{p\left(\lambda-q r \mu_{v}+\gamma\right)}{(1-p)(1-\beta)}+\frac{\lambda \alpha}{(1-\rho)(1-r)} \\
& \left.+\frac{\left(\lambda-q r \mu_{v}+\gamma\right) \rho}{1-\rho}+\frac{p \rho\left(\lambda-q r \mu_{v}+\gamma\right)}{(1-p)(1-\beta)(1-\rho)}\right] \\
\left(K^{*}\right)^{-1}= & \left.\frac{\lambda}{r}+\frac{p\left(\lambda-q r \mu_{v}+\gamma\right)}{1-p}\right](1-r)(1-\beta) \\
& +\left[\rho\left(\lambda-q r \mu_{v}+\gamma\right)-\frac{\lambda \alpha}{r}\right](1-r)(1-\beta) \\
& +(1-\beta)\left[\frac{\lambda}{r}(r-\rho)+\frac{\lambda \alpha}{r}\right]+(1-r) \\
& {\left[\frac{p\left(\lambda-q r \mu_{v}+\gamma\right)(\beta-\rho)}{1-p}+\frac{\rho p\left(\lambda-q r \mu_{v}+\gamma\right)}{1-p}\right] }
\end{aligned}
$$

The above equation indicates.

$Q(z)=\frac{1-\rho}{1-\rho z} Q_{d}(z)$

is a probability generating function. Expanding $Q_{d}(z)$ into power series of $z$. We get the distribution of additional number of customers $Q_{d}$.

With the stochastic decomposition structure in theorem 4,we can easily get means. 


$$
\begin{aligned}
E\left(Q_{d}\right)= & K^{*}\left[\left[\rho\left(\lambda-q r \mu_{v}+\gamma\right)-\frac{\lambda \alpha}{r}\right](1-r)(1-\beta)\right. \\
& +(1-\beta)\left[\frac{\lambda}{r}(r-\rho)+\frac{\lambda \alpha}{r}\right] \frac{1}{1-r} \\
& +(1-r)\left[\frac{p\left(\lambda-q r \mu_{v}+\gamma\right)(\beta-\rho)}{1-p}\right. \\
\left.\left.+\frac{\rho p\left(\lambda-q r \mu_{v}+\gamma\right)}{1-p}\right] \frac{1}{1-\beta}\right] & 1-p \\
E(Q)= & \frac{\rho}{1-\rho}+E\left(Q_{d}\right)
\end{aligned}
$$

\section{Theorem 4.}

If $\rho<1$ and $\mu_{b}>\mu_{v}$, the stationary waiting time $\mathrm{W}$ of an arrival can be decomposed into the sum of two independent variables: $W=W_{0}+W_{d}$, where $W_{0}$ is the waiting time of an arrival in a corresponding classical $\mathrm{M} / \mathrm{M} / 1$ queue and is exponentially distributed with parameter $\mu_{b}(1-\rho)$ and $W_{d}$ is the additional delay with the LST given by

$$
\begin{aligned}
& W_{d}^{*}(s)=K^{*}\left[\left\{\frac{\lambda}{r}+\frac{p\left(\lambda-q r \mu_{v}+\gamma\right)}{1-p}+\rho\left(\lambda-q r \mu_{v}+\gamma\right)-\frac{\lambda \alpha}{r}\right.\right. \\
& -\frac{1}{r}\left[\frac{\lambda}{r}(r-\rho)+\frac{\lambda \alpha}{r}\right]-\frac{1}{\beta}\left[\frac{p\left(\lambda-q r \mu_{v}+\gamma\right)(\beta-\rho)}{1-p}\right. \\
& \left.\left.+\frac{\rho p\left(\lambda-q r \mu_{v}+\gamma\right)}{1-p}\right]\right\}(1-r)(1-\beta) \\
& -\left(\frac{p\left(\lambda-q r \mu_{v}+\gamma\right)}{1-p}+\rho\left(\lambda-q r \mu_{v}+\gamma\right)\right. \\
& \left.-\frac{\lambda \alpha}{r}\right) \frac{(1-r)(1-\beta)}{\lambda}+\frac{1-\beta}{r} \\
& \left(\frac{\lambda}{r}(r-\rho)+\frac{\lambda \alpha}{r}\right) \frac{\frac{\lambda}{r}-\lambda}{\frac{\lambda}{r}-\lambda+s} \\
& -\frac{1-r}{\beta}\left[\frac{p\left(\lambda-q r \mu_{v}+\gamma\right)(\beta-\rho)}{1-p}\right. \\
& \left.\left.+\frac{\rho p\left(\lambda-q r \mu_{v}+\gamma\right)}{1-p}\right] \frac{\frac{\lambda}{\beta}-\lambda}{\frac{\lambda}{\beta}-\lambda+s}\right]
\end{aligned}
$$

\section{Proof.}

The classical relation between the probability generating function of L and LST of waiting time W (Keilsen and servi) is $Q(z)=W^{*}(\lambda(1-z))$

From theorem 3 , the probability generating function of the number of customers Q can be written as,

$$
\begin{aligned}
& Q(z)=\frac{1-\rho}{1-\rho z} K^{*}\left[\left(\frac{\lambda}{r}+\frac{p\left(\lambda-q r \mu_{v}+\gamma\right)}{1-p}\right)(1-r)(1-\beta)\right. \\
& +\left[\rho\left(\lambda-q r \mu_{v}+\gamma\right)-\frac{\lambda \alpha}{r}\right](1-r)(1-\beta) z \\
& +(1-\beta)\left[\frac{\lambda}{r}(r-\rho)+\frac{\lambda \alpha}{r}\right] \frac{1-r}{1-r z} z \\
& +(1-r)\left[\frac{p\left(\lambda-q r \mu_{v}+\gamma\right)(\beta-\rho)}{1-p}\right. \\
& \left.\left.+\frac{\rho p\left(\lambda-q r \mu_{v}+\gamma\right)}{1-p}\right] \frac{1-\beta}{1-\beta z} z\right]
\end{aligned}
$$

Taking $z=1-\frac{s}{\lambda}$ in (12), we get

$$
\frac{1-\rho}{1-\rho z}=\frac{\mu_{b}(1-\rho)}{\mu_{b}(1-\rho)+s}, \frac{1-r}{1-r z}=\frac{\frac{\lambda}{r}-\lambda}{\frac{\lambda}{r}-\lambda+s},
$$

$$
\frac{1-\beta}{1-\beta z}=\frac{\frac{\lambda}{\beta}-\lambda}{\frac{\lambda}{\beta}-\lambda+s}
$$

Substituting the above result into (12), we get

$$
\begin{aligned}
& W^{*}(s)=\frac{\mu_{b}(1-\rho)}{\mu_{b}(1-\rho)+s} K^{*}\left\{\left(\frac{\lambda}{r}+\frac{p\left(\lambda-q r \mu_{v}+\gamma\right)}{1-p}\right)\right. \\
& (1-r)(1-\beta)+\left[\rho\left(\lambda-q r \mu_{v}+\gamma\right)\right. \\
& \left.-\frac{\lambda \alpha}{r}\right](1-r)(1-\beta)\left(1-\frac{s}{\lambda}\right)-\frac{(1-r)(1-\beta)}{r} \\
& {\left[\frac{\lambda}{r}(r-\rho)+\frac{\lambda \alpha}{r}\right]+\frac{(1-\beta)}{r}\left[\frac{\lambda}{r}(r-\rho)+\frac{\lambda \alpha}{r}\right]} \\
& \frac{\frac{\lambda}{r}-\lambda}{\frac{\lambda}{r}-\lambda+s}-\frac{(1-\beta)(1-r)}{\beta} \\
& {\left[\frac{p\left(\lambda-q r \mu_{v}+\gamma\right)(\beta-\rho)}{1-p}+\frac{\rho p\left(\lambda-q r \mu_{v}+\gamma\right)}{1-p}\right]} \\
& +\frac{(1-r)}{\beta}\left[\frac{p\left(\lambda-q r \mu_{v}+\gamma\right)(\beta-\rho)}{1-p}\right. \\
& \left.\left.+\frac{\rho p\left(\lambda-q r \mu_{v}+\gamma\right)}{1-p}\right] \frac{\frac{\lambda}{\beta}-\lambda}{\frac{\lambda}{\beta}-\lambda+s}\right\} \\
& W_{d}^{*}(s)=K^{*}\left[\left\{\frac{\lambda}{r}+\frac{p\left(\lambda-q r \mu_{v}+\gamma\right)}{1-p}\right.\right. \\
& +\rho\left(\lambda-q r \mu_{v}+\gamma\right)-\frac{\lambda \alpha}{r}-\frac{1}{r}\left[\frac{\lambda}{r}(r-\rho)+\frac{\lambda \alpha}{r}\right]
\end{aligned}
$$


$\left.-\frac{1}{\beta}\left[\frac{p\left(\lambda-q r \mu_{v}+\gamma\right)(\beta-\rho)}{1-p}+\frac{\rho p\left(\lambda-q r \mu_{v}+\gamma\right)}{1-p}\right]\right\}(1-r)(1-\beta)$

$-\left(\frac{p\left(\lambda-q r \mu_{v}+\gamma\right)}{1-p}+\rho\left(\lambda-q r \mu_{v}+\gamma\right)-\frac{\lambda \alpha}{r}\right) \frac{(1-r)(1-\beta)}{\lambda}$

$+\frac{1-\beta}{r}\left(\frac{\lambda}{r}(r-\rho)+\frac{\lambda \alpha}{r}\right) \frac{\frac{\lambda}{r}-\lambda}{\frac{\lambda}{r}-\lambda+s}$

$\left.-\frac{1-r}{\beta}\left[\frac{p\left(\lambda-q r \mu_{v}+\gamma\right)(\beta-\rho)}{1-p}+\frac{\rho p\left(\lambda-q r \mu_{v}+\gamma\right)}{1-p}\right] \frac{\frac{\lambda}{\beta}-\lambda}{\frac{\lambda}{\beta}-\lambda+s}\right]$

The proof the theorem 4, we give the equivalent expression of $K *$

$$
\begin{aligned}
\left(K^{*}\right)^{-1}= & {\left[\frac{\lambda}{r}+\frac{p\left(\lambda-q r \mu_{v}+\gamma\right)}{1-p}\right](1-r)(1-\beta) } \\
& +\left[\rho\left(\lambda-q r \mu_{v}+\gamma\right)-\frac{\lambda \alpha}{r}\right](1-r)(1-\beta) \\
& +(1-\beta)\left[\frac{\lambda}{r}(r-\rho)+\frac{\lambda \alpha}{r}\right] \\
& +(1-r)\left[\frac{p\left(\lambda-q r \mu_{v}+\gamma\right)(\beta-\rho)}{1-p}+\frac{\rho p\left(\lambda-q r \mu_{v}+\gamma\right)}{1-p}\right]
\end{aligned}
$$

Hence, this proves that $W_{d}^{*}(s)$ is a LST.

Based on the above stochastic decomposition structure, we get easily obtain the means as follows

$$
\begin{aligned}
E\left(W_{d}\right) & =\frac{1}{\lambda} K^{*}\left\{\left[\rho\left(\lambda-q r \mu_{v}+\gamma\right)-\frac{\lambda \alpha}{r}\right](1-r)(1-\beta)\right. \\
& +(1-\beta)\left[\frac{\lambda}{r}(r-\rho)+\frac{\lambda \alpha}{r}\right] \\
& \left.+(1-r)\left[\frac{p\left(\lambda-q r \mu_{v}+\gamma\right)(\beta-\rho)}{1-p}+\frac{\rho p\left(\lambda-q r \mu_{v}+\gamma\right)}{1-p}\right]\right\} \\
& =\frac{1}{\lambda} E\left(Q_{d}\right) \\
E(W) & =\frac{1}{\mu_{b}(1-\rho)}+E\left(W_{d}\right)
\end{aligned}
$$

Numerical Results: In this section, we illustrate the influence of the system parameters on the performance measures by presenting some numerical examples. The various parameters of the model are chosen as $\lambda=1, \mu=2, \eta=1, \theta=0.6, p=0.3$ and $q=0.5$ The main findings in this study are itemized as

- Fig. 1 presents the effects of $\eta$ on E[L] for different values of $\gamma$. Clearly, $\mathrm{E}[\mathrm{L}]$ decreases as $\eta$ increases. This is due to the fact that larger the service rate $\eta$ during working vacation, higher is the probability that the customers are served during this period which leads to the decrease in $\mathrm{E}[\mathrm{L}]$. Also, the queue length increases as we increase the value of $\gamma$.
- Fig. 2 presents the effects of $\mu$ on E [L] without VI (q=0) and with Vacation Interruptions ( $\mathrm{q}=1)$. As expected, for any $\mathrm{q}, \mathrm{E}[\mathrm{L}]$ deceases with the increase of normal service rate $\mu$. It is observed that models without VI $(\mathrm{q}=0)$ gives higher queue lengths as compared to models with VI $(\mathrm{q}=1)$. This is because of the fact that in models without VI, the server stays in working vacation for the entire duration of the vacation which resulted in the increase in queue length $\mathrm{E}[\mathrm{L}]$.

- Figs. 3 and 4 presents the state probability of the server and the probability that the server remains in normal working period, i.e., $\mathrm{P}(\mathrm{J}=2)$, evidently decreases with the increase in $\eta$. The probability that the server remains in working vacation period $\mathrm{P}(\mathrm{J}=0)$ increases, thus, the utilization level of the system idle time also become larger. Note that the working vacation rate $\gamma$ also has some impact on the state probability of the server. For instance, when $\gamma=1.5, \mathrm{P}(\mathrm{J}=2)$ are evidently smaller when compared to $\gamma=0.5$.

- Fig 5. illustrates the impact of arrival rate $\lambda$ on mean queue length $\mathrm{E}[\mathrm{L}]$. As expected, $\mathrm{E}[\mathrm{L}]$ increases with the increase in $\lambda$.

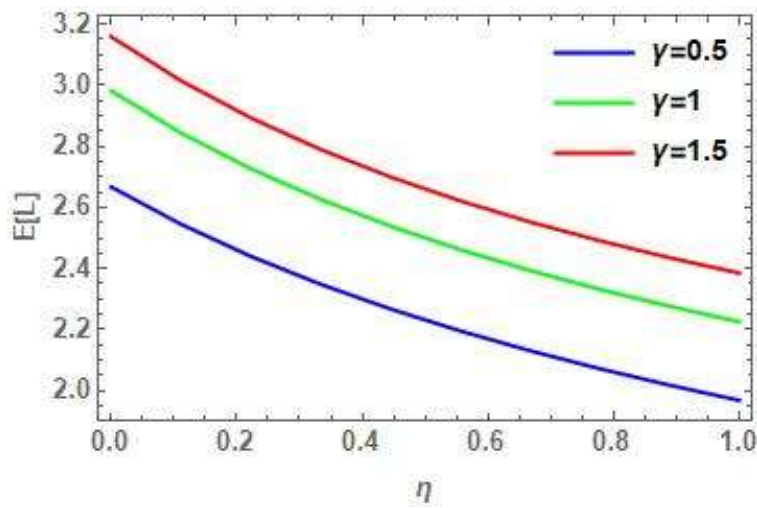

Figure 1: Impact of $\eta$ on $\mathrm{E}(\mathrm{L})$

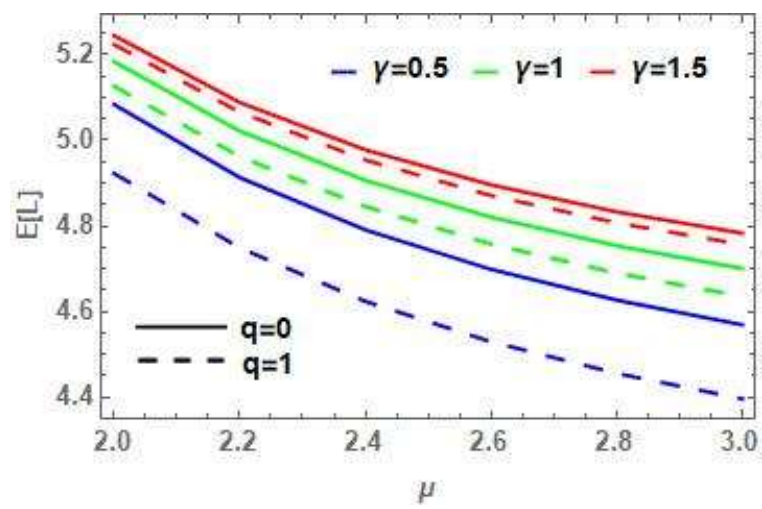

Figure 2: Impact of $\mu$ on $\mathrm{E}(\mathrm{L})$

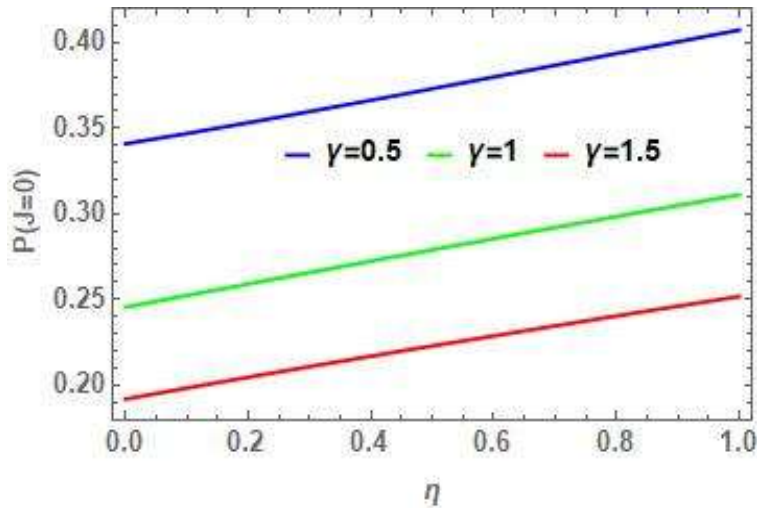

Figure 3: The state probability of the server against $\eta$ 


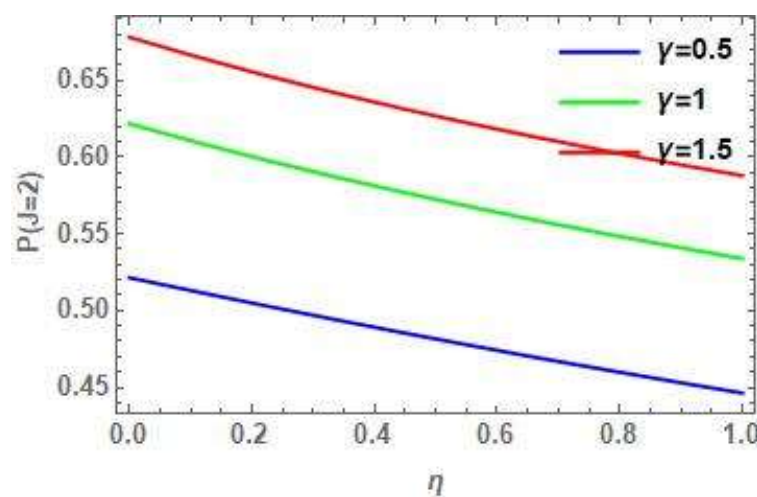

Figure 4: The state probability of the server against $\eta$

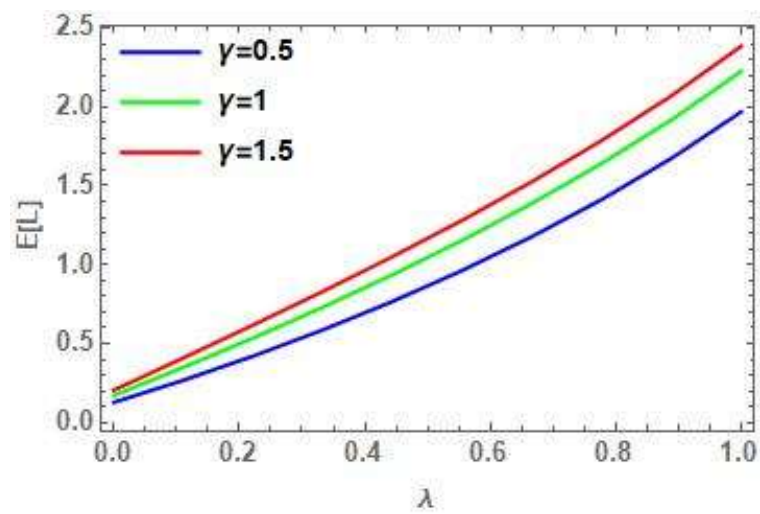

Figure 5: Impact of $\lambda$ on $\mathrm{E}[\mathrm{L}]$

\section{References}

[1] Baba, Y. (2005). Analysis of a GI/M/1 queue with multiple working vacations, Operations Research Letters, 33: 201-209.

[2] Baba, Y. (2010) The M/PH/1 queue with working vacations and vacation interruption, Journal of Systems Science and Systems Engineering 19(4): 496-503

[3] Banik, A. (2010). Analysis of single server working vacation in $\mathrm{GI} / \mathrm{M} / 1 / \mathrm{N}$ and $\mathrm{GI} / \mathrm{M} / 1 / 1$ queueing systems, International Journal of Operational Research, 7(3): 314-333.

[4] Chao, X. and Zhao, Y.Q. (1998). Analysis of multiserver queues with station and server vacations, European Journal of Operational Research, 110(2): 392-406

[5] Doshi, B.T. (1986). Queueing systems with vacations a survey, Queueing Systems, 1: 29-66.

[6] Gao, S. and Liu, Z. (2013). An M/G/1 queue with single working vacation and vacation interruption under Bernoulli schedule, Applied Mathematical Modelling 37: 1564-1579,

[7] Ke, J., Wu, C. and Zhang, Z. (2010). Recent developments in vacation queueing models, A short survey, International Journal of Operations Research, 7(4): 3-8.

[8] Laxmi, P. and Yesuf, O. (2011). Renewal input infinite buffer batch service queue with single exponential working vacation and accessibility to batches, International, Journal of Mathematics in Operational Research, 3(2): 219-243.

[9] Lee, D.H. and Kim, B.K. (2015). A note on the sojourn time distribution of an M/G/1 queue with a single working vacation and vacation interruption, Operations Research Perspectives 2, 57-61.

[10] Levy, Y. and Yechiali, U. (1976). An M/M/c queue with servers vacations, INFOR, 14(2): 153-163.

[11] Li, J.H. and Tian, N.S. (2007) The M/M/1 queue with working vacations and vacation interruptions, Journal of Systems Science and Systems Engineering 16(1): 121-127.

[12] Li, J.H. Tian, N.S. and Ma, Z.Y. (2008) Performance analysis of $\mathrm{GI} / \mathrm{M} / 1$ queue with working vacation and vacation interruption, Applied Mathematical Modelling 32(12),2715-2730.

[13] Lin, C.H and Ke, J.C. (2009). Multi-server system with single working vacation, Applied Mathematical Modelling, 33:2967-2977.

[14] Liu, W., Xu, X. and and Tian, N. (2007). Stochastic decomposition in the $\mathrm{M} / \mathrm{M} / 1$ queue with working vacations, Operations Research Letters, 35: 595-600.
[15] Servi, L.D. and Finn, S.G. (2002). M/M/1 queues with working vacations (M/M/1/WV), Perform. Eval. 50: 41-52.

[16] Shakir Majid, and Manoharan. P (2017), Analysis of the M/M/1 queue with single working vacation and vacation interruption, International journal of mathematics trends and technology, vol-47, 31-39.

[17] Takagi, H. (1991). Queueing Analysis “ A Foundation of Performance Evaluation Vacation and Priority Systems, vol.1: NorthHolland, New York,

[18] Wu, D.A. and Takagi, H. (2006). M/G/1 queue with multiple working vacations, Performance Evaluation, 63(7): 654-681.

[19] Zhang, M. and Hou, M. (2011). Performance analysis of MAP/G/1 queue with working vacations and vacation interruption, Applied Mathematical Modelling 35(4): 1551-1560.

[20] Zhang, Z.G. and Tian, N. (2003a). Analysis of queueing systems with synchronous single vacation for some servers, Queueing Systems, 45: 161-175.

[21] Zhang, Z.G. and Tian, N. (2003b). Analysis on queueing systems with synchronous vacations of partial servers, Perform. Eval. 52: $269-282$ 